Geosci. Model Dev., 11, 1405-1420, 2018

https://doi.org/10.5194/gmd-11-1405-2018

(C) Author(s) 2018. This work is distributed under

the Creative Commons Attribution 4.0 License.

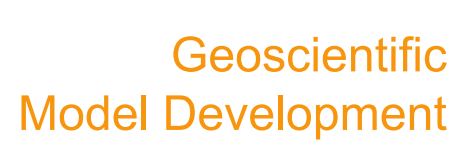

(c) (i)

\title{
OpenDrift v1.0: a generic framework for trajectory modelling
}

\author{
Knut-Frode Dagestad ${ }^{1}$, Johannes Röhrs ${ }^{1}, \emptyset_{\text {yvind Breivik }}^{1,3}$, and Bjørn Ådlandsvik ${ }^{2}$ \\ ${ }^{1}$ Norwegian Meteorological Institute, Bergen, Norway \\ ${ }^{2}$ Institute of Marine Research, Bergen, Norway \\ ${ }^{3}$ Geophysical Institute, University of Bergen, Bergen, Norway
}

Correspondence: Knut-Frode Dagestad (knutfd@met.no)

Received: 20 August 2017 - Discussion started: 30 August 2017

Revised: 6 February 2018 - Accepted: 19 February 2018 - Published: 13 April 2018

\begin{abstract}
OpenDrift is an open-source Python-based framework for Lagrangian particle modelling under development at the Norwegian Meteorological Institute with contributions from the wider scientific community. The framework is highly generic and modular, and is designed to be used for any type of drift calculations in the ocean or atmosphere. A specific module within the OpenDrift framework corresponds to a Lagrangian particle model in the traditional sense. A number of modules have already been developed, including an oil drift module, a stochastic search-and-rescue module, a pelagic egg module, and a basic module for atmospheric drift. The framework allows for the ingestion of an unspecified number of forcing fields (scalar and vectorial) from various sources, including Eulerian ocean, atmosphere and wave models, but also measurements or a priori values for the same variables. A basic backtracking mechanism is inherent, using sign reversal of the total displacement vector and negative time stepping. OpenDrift is fast and simple to set up and use on Linux, Mac and Windows environments, and can be used with minimal or no Python experience. It is designed for flexibility, and researchers may easily adapt or write modules for their specific purpose. OpenDrift is also designed for performance, and simulations with millions of particles may be performed on a laptop. Further, OpenDrift is designed for robustness and is in daily operational use for emergency preparedness modelling (oil drift, search and rescue, and drifting ships) at the Norwegian Meteorological Institute.
\end{abstract}

\section{Introduction}

Lagrangian trajectory models are used to predict the pathways and transformations of various types of objects and substances drifting in the ocean or in the atmosphere. There are many practical and academic applications, including prediction of

- oil drift and weathering to aid mitigation and cleanup operations (Jones et al., 2016);

- drifting objects for search and rescue (Breivik and Allen, 2008; Breivik et al., 2011, 2013);

- ichthyoplankton transport (fish eggs and larvae) for stock assessments (Röhrs et al., 2014); and

- microplastics suspended in the ocean (van Sebille et al., 2012, 2015).

Table 1 lists some commonly used trajectory models and their applications. Additionally, many individual researchers or research groups have been developing trajectory model codes for in-house use, without publishing (or naming) a software code.

Lagrangian tools fall in two broad categories: either the trajectories are computed along with the velocity fields as part of the ocean or atmospheric circulation model, e.g. socalled floats in the regional ocean modelling system (ROMS) (Shchepetkin and McWilliams, 2005). This is known as "online" trajectory computations and has the advantage that no separate model is needed. Alternatively, the trajectories can be computed "offline" after completion of the Eulerian model simulation(s). This is the approach taken for OpenDrift and is also necessary for a generic framework as the trajectories 
depend in many cases on forcing from a range of fields stemming from more than just one Eulerian model. This is, e.g. the case for oil drift and search-and-rescue models, which both require wind as well as currents (and wave forcing in the case of oil drift) to properly account for the advection and transformation of the particles. For such emergency preparedness purposes, offline models are the only option fast enough to meet the requirements of operational agencies. Other advantages of offline models are that modifications (sensitivity tests) of the drift algorithms may be tested quickly without needing to rerun the full Eulerian model, and also that simulations backwards in time may be performed.

Existing trajectory models are in most cases tied to a specific application, and may not be applied to other drift applications without compromising quality or flexibility. In many cases, trajectory models are also tied to a specific Eulerian model, or even a particular institutional ocean model setup, limiting usability for other institutes or researchers. Often, it is also required that Eulerian forcing data must be in a specific file format. This raises the time and effort needed to set up a trajectory model. Further, in an operational setup, the need to convert large files to another format increases both the complexity and computational costs of the processing chain, compromising robustness.

The OpenDrift framework has been designed to perform all tasks which are common to trajectory models, whether oceanic or atmospheric. In short, the main task is to obtain forcing data from various sources and to use this information to move (propagate) the elements in space, while potentially transforming other element properties, such as evaporation of oil, or growth of larvae. In addition, common functionality includes mechanisms for configuration of simulations, seeding of elements, exporting output to file, and tools to visualise and analyse the output. Additionally, several design requirements have been imposed on the development of OpenDrift: (1) platform independence and ease of installation and use; (2) simple and rapid implementation of any purpose-specific processes, yet flexibility to support unforeseen needs; (3) forcing data from any type of source supported, including Eulerian ocean, atmosphere or wave models (typically NetCDF or GRIB files), in situ measurements, vector data sets (e.g. GSHHS coastlines) or analytical fields for conceptual studies; (4) the ability run fast, even with a large number of elements; (5) simulations forward and backward in time; (6) robustness for operational use.

In Sect. 2, we describe the overall design of the code and the general workflow of performing a simulation. In Sect. 3, we give examples of three specific modules which are included in the OpenDrift repository: search and rescue, oil drift and atmospheric transport. The test suite and example scripts are described in Sect. 4, and graphical user interfaces (web and desktop) are described in Sect. 5. Section 6 provides discussion and conclusions.

\section{Software design}

To meet the requirements listed above, a simple and flexible object-oriented data model has been designed, based on two main classes. One class ("Reader") is dedicated to obtaining forcing data from external sources, as described in Sect. 2.1. A generic class for a trajectory model instance ("BaseModel") is described in Sect. 2.2. This class contains functionality which is common to all drift models, whereas advection (propagation) and transformation of elements is left for purpose-specific subclasses.

\section{$2.1 \quad$ Reader class}

The class Reader obtains forcing data (e.g. wind, waves and currents) from any possible source and provides this to any OpenDrift model through a common interface. To avoid duplication of code, a parent class "BaseReader" contains functionality which is common to all Readers, whereas specific subclasses take care of only the tasks which are specific to a particular source of data, e.g. how to decode and interpret a particular file format. Two methods must be implemented by any Reader subclass: (1) a constructor method which initialises a Reader object and (2) a method to retrieve data for given variables, position and time. The constructor (__init__ in Python) can take any arguments as implemented by the specific Reader class, but it is typical to provide a filename or URL from which data shall be obtained by this Reader. The following Python commands initialise a Reader of type NetCDF_CF_generic to obtain data from a file "ocean_model_output.nc".

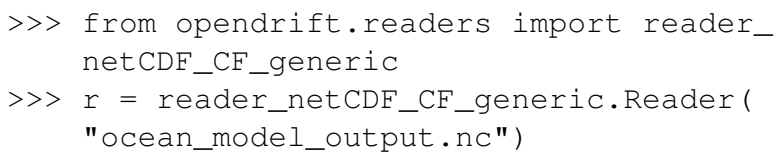

The initialisation typically includes opening and reading metadata from a given file or URL to check which variables are available, and the coverage in time and space. The actual reading of the data is, however, not performed yet, but is delayed until it is known exactly which subset in space and time is actually needed ("lazy reading"). The contents can be inspected by printing the object:

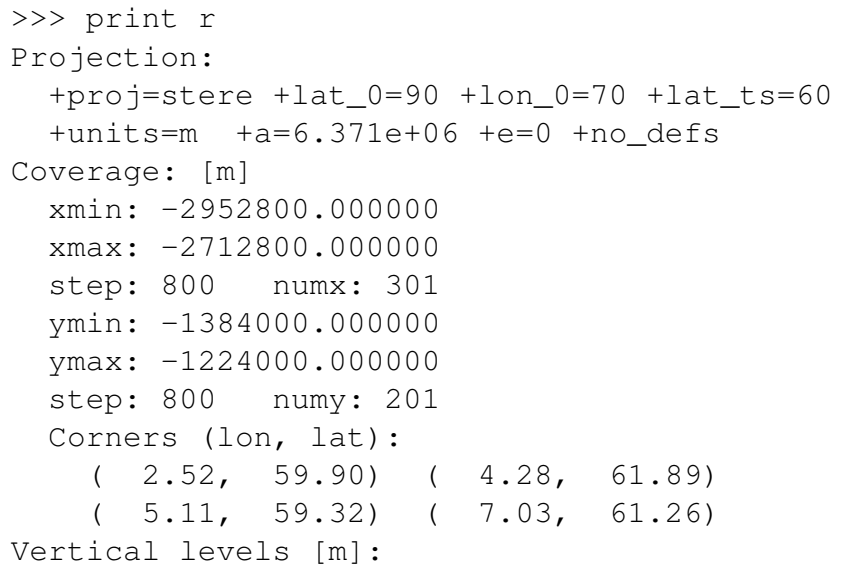


Table 1. Some existing trajectory models for various oceanic and atmospheric applications.

\begin{tabular}{|c|c|c|}
\hline Name & Reference/URL & Main application \\
\hline Ariane & Blanke et al. (1997) & Oceanography \\
\hline BSHDmod & Dick and Soetje (1990) & Oil \\
\hline Connectivity Modeling System & Paris et al. (2013) & Ocean, generic \\
\hline CIS iceberg model & Kubat et al. (2007) & Icebergs \\
\hline CLaMS & McKenna et al. (2002) & Atmospheric chemistry \\
\hline EMEP & Simpson et al. (2012) & Air pollution \\
\hline FLEXPART, FLEXSTRA & $\begin{array}{l}\text { www.flexpart.eu, } \\
\text { Stohl et al. (1995) }\end{array}$ & Nuclear, air pollution \\
\hline HYSPLIT & Stein et al. (2015) & Atmospheric transport \\
\hline Ladim & Ådlandsvik and Sundby (1994) & Plankton transport \\
\hline LAGRANTO & $\begin{array}{l}\text { Wernli and Davies (1997), } \\
\text { Sprenger and Wernli (2015) }\end{array}$ & Meteorology \\
\hline LAGRANTO.ocean & Schemm et al. (2017) & Water mass properties \\
\hline Leeway & $\begin{array}{l}\text { Breivik and Allen (2008), } \\
\text { Allen and Plourde (1999) }\end{array}$ & Search and rescue \\
\hline LTRANS & Schlag and North (2012) & Plankton (including larvae) \\
\hline MEDSLIK, MEDSLIK-II & $\begin{array}{l}\text { De Dominicis et al. (2013), } \\
\text { Lardner et al. (1998) }\end{array}$ & Oil \\
\hline MIKE & www.mikepoweredbydhi.com & Ocean, generic \\
\hline MOHID & www.mohid.com & Oil, sediments, water quality \\
\hline MOTHY & Daniel (1996) & Oil, drifting objects \\
\hline OD3D & Wettre et al. (2001) & Oil \\
\hline $\begin{array}{l}\text { OILMAP, SIMAP, CHEMMAP, } \\
\text { MUDMAP, SARMAP }\end{array}$ & www.asascience.com & $\begin{array}{l}\text { Oil, sediments, chemical, search } \\
\text { and rescue }\end{array}$ \\
\hline OILTOX & Brovchenko et al. (2003) & Oil \\
\hline OILTRANS & Berry et al. (2012) & Oil \\
\hline OSCAR & www.sintef.no/en/software/oscar & Oil \\
\hline OSERIT & oserit.mumm.ac.be & Oil, chemicals \\
\hline PARCELS & https://github.com/OceanPARCELS/parcels & Ocean, generic \\
\hline POSEIDON-OSM & osm.hemr.gr & Oil \\
\hline PyGNOME/GNOME & gnome.orr.noaa.gov & Oil, generic \\
\hline SeaTrackWeb, PADM & stw.smhi.se & Oil, chemicals \\
\hline SNAP & Bartnicki et al. (2016) & Atmospheric nuclear transport \\
\hline STILT & www.stilt-model.org & Atmospheric trace gases \\
\hline THREETOX & Margvelashvily et al. (1997) & Nuclear ocean transport \\
\hline TRACMASS & Döös et al. (2013) & Ocean and atmosphere, generic \\
\hline VOS & en.ferhri.org & Oil \\
\hline
\end{tabular}

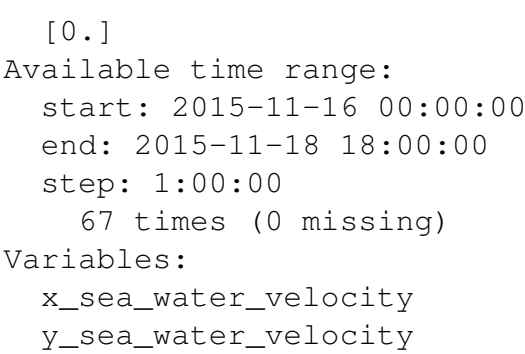

The above example shows that the created Reader object can provide ocean surface current on a grid with $800 \mathrm{~m}$ pixel size in polar stereographic coordinates, at hourly time resolution.

To allow for generic coupling of any OpenDrift model with any Reader, a naming convention for variables is necessary. By convention, the commonly used Climate and Fore- cast (CF) naming convention (cfconventions.org) should be used whenever possible. Thus, if the data source is not already following this convention (e.g. a GRIB file), the Reader should map the variable names to corresponding CF standard_name.

The given Reader class must also have implemented a specific instance of the method get_variables which is called to return data:

$$
\begin{aligned}
>>\text { data }= & \text { r.get_variables (["x_wind", } \\
& \text { "y_wind"], } x, y, z, \text { time) }
\end{aligned}
$$

The horizontal coordinates $(\mathrm{x}, \mathrm{y})$ correspond to the native projection of the Reader, which is polar stereographic in the given example. The task of transforming from one coordinate system to another (including the rotation of vectors) is performed by common methods from the parent class, based on 
the widely used PROJ.4 library (proj4.org), through its Python interface pyproj. This allows OpenDrift to combine input data from any coordinate systems, whilst keeping the implementation of new Reader classes as minimalistic and clean as possible. Further, this centralisation of code also facilitates optimisation for both performance and robustness. The vertical coordinate $(z)$ is by convention always in metres, zero at the air/water surface and positive upwards. Thus, Readers providing data from sources with other vertical coordinate systems (e.g. topography following coordinates or pressure/density levels) must take care of transforming this to metres before data are returned. This is, e.g. done by an existing Reader supporting native output from the ROMS ocean model. The variable time is consistently handled as Python datetime objects within OpenDrift, with any time intervals as timedelta objects. Readers also share some common convenience methods, such as plotting of geographical coverage.

Readers are, however, normally not called directly by the user or from specific OpenDrift instances (models) but rather implicitly from the parent BaseModel class (see Sect. 2.2). An internal caching mechanism is implemented to minimise the amount of data to be read, which is key to improving performance. Input data from numerical models are normally provided on a 3-D spatial grid $(x, y, z)$ at discrete time steps, which are often larger than the time steps used internally by OpenDrift models. When data are requested for a given set of element positions at a given time, OpenDrift requests from the Readers' 3-D blocks of data from the time before and after the given time. These 3-D blocks encompass the elements tightly, except for a buffer on each side which is large enough so that elements will stay within the coverage during the time step of the Reader. After 3-D blocks of data are provided by the Reader, interpolators are generated and then reused to interpolate the same data blocks onto the element positions successively at each internal calculation time step, until the calculation time step reaches the latter Reader (model) time step. At this point, a 3-D block for the subsequent model time step is requested, and a new interpolator is generated. Due to this very economical access of remote data, simulations with OpenDrift are almost as fast when obtaining data from remote Thredds servers, as when reading the same data from a local file. The interpolator mechanism is also modularised by a dedicated class in OpenDrift, allowing independent development and optimisation. The default interpolation algorithm uses bilinear interpolation (scipy.ndimage.map_coordinates) and may also extrapolate data towards land, to avoid particles stranding in a "no data" gap between ocean pixels from an ocean model and land points as determined from an independent land mask.

Functionality exists also for reading and interpolating data from ensemble models. For example, when obtaining wind from a NetCDF model file containing 10 ensemble members, particles number $1,11,21 \ldots$ will use wind from member 1 of the atmospheric ensemble, and particles number $2,12,22 \ldots$ will use wind from member 2 of the atmospheric ensemble and so on. This allows for a more realistic spread/diffusion of particles than when using no or constant diffusivity. This functionality is particularly useful for ocean model output, which is inherently uncertain on short timescales, due to limited availability of observations for assimilation.

Whereas obtaining forcing data from 3-D Eulerian models is the most common in practice, Readers may obtain data from any other possible source. One example is to read a time series from an American Standard Code for Information Interchange (ASCII) file of observations, e.g. from a buoy or a weather station. Another example is to calculate forcing data according to some analytical function. One such example is included in the code repository, providing ocean current vectors according to a "perfect circular eddy" with centre coordinates as given to its constructor. Such analytical forcing data fields are useful for, e.g. testing the accuracy of forward propagation schemes, as discussed below.

OpenDrift also contains some internal convenience methods to calculate geophysical variables from others. For example, if a drift module requires wave height or Stokes drift, this may be parameterised internally based on the wind velocity if no Readers providing wave parameters are available.

\subsection{BaseModel class}

Functionality which is common to any trajectory model is described in a main class, named BaseModel. This functionality includes the following:

1. A mechanism for configuration of a trajectory model, or a specific simulation is needed. This may include adjusting the resolution of a coastline or some modelspecific parameters concerning the movement of the elements. The configuration mechanism of OpenDrift is based upon the ConfigObj package (https://pypi.python. org/pypi/configobj).

2. A generic method to seed elements for a simulation is required. See Sect. 2.3.3 for details.

3. Managing and referencing a set of Readers (Sect. 2.1), which are called as needed to obtain forcing data during a simulation, are also necessary. See Sect. 2.3.2 for details.

4. It is required to keep track of the positions and properties of all elements during a simulation and remove elements scheduled for deactivation. This is stored in 2-D arrays with two dimensions, time and particle ID. Thus, the trajectory (propagation with time) of a single element or the simulation state (all element positions and properties at a given time) is easily and quickly obtained as vertical or horizontal slices of the array. The history of data may also be written to file, as described in Sect. 2.3.6. 


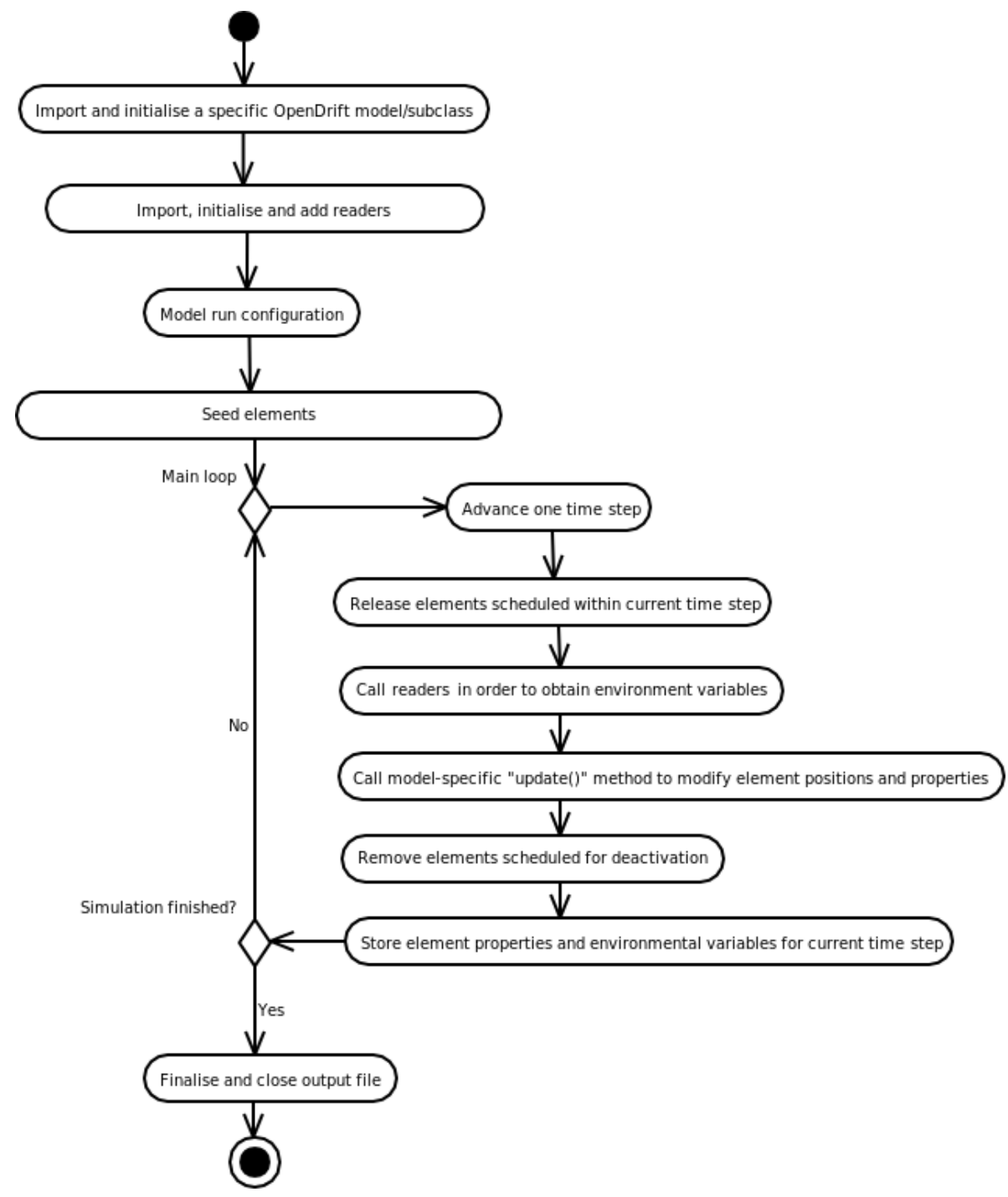

Figure 1. Flowchart of an OpenDrift simulation.

5. Finally, the BaseModel class contains the main loop for time stepping, performing necessary tasks for a simulation in the correct order, as described in Sect. 2.3.5.

The only part missing is a description of how the elements (e.g. objects or substance) shall be propagated and potentially transformed along their trajectories under the influence of environmental forcing data. Such application-specific description is left to subclasses, yielding trajectory model instances as exemplified in Sect. 3. These subclasses thus inherit and may reuse any functionality from the BaseModel. The subclasses may also add further functionality as needed, or overload and modify existing functionality. Thus, all necessary core functionality is available by convenience but may be modified for flexibility. In precise terminology, OpenDrift is a framework within which specific trajectory models may be implemented by class inheritance (subclassing). An in- stance (object) of such a subclass represents a specific trajectory simulation.

\subsection{Performing a simulation}

In this section, we describe and explain the general workflow of a simulation with an OpenDrift model, as illustrated in the flowchart in Fig. 1.

\subsubsection{Initialisation}

The first step is to import a specific OpenDrift model (subclass of BaseModel) and to initialise an instance. The following Python statements import and initialise an instance of the Leeway search-and-rescue model (Sect. 3.1). 


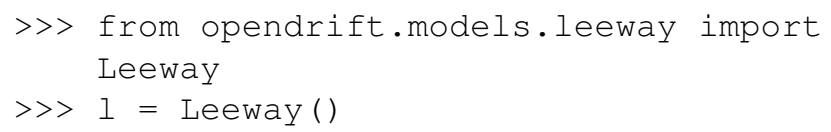

\subsubsection{Adding Readers}

If a given model requires, e.g. ocean current and atmospheric wind as environmental forcing, we need to create and add Reader instances which can provide these variables. Say that we have a Thredds server which can provide ocean currents and a local GRIB file which contains atmospheric winds; we can create and add these Readers to the simulation instance as follows:

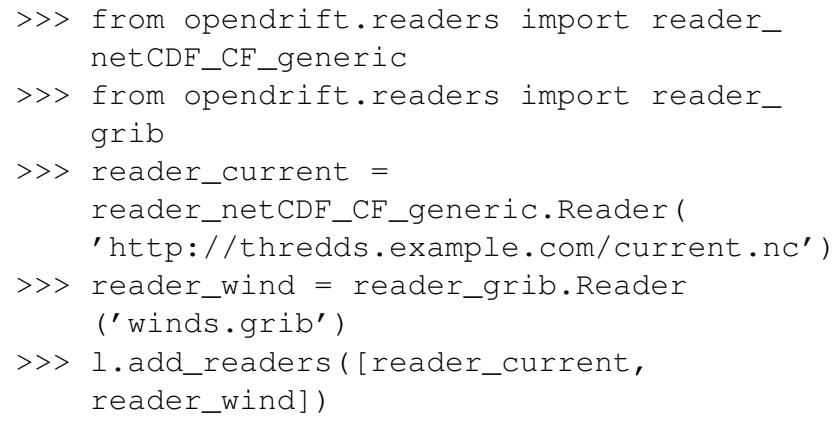

For NetCDF files, it is also possible to create a single Reader object which merges together many files by using wildcards (* or ?) in the filename. This functionality is based on the NetCDF MFDataset class.

It is also possible to perform a simulation even with no Readers added for one or more of the required variables. In this case, constant values may be provided; otherwise, reasonable default values will be used, defaulting to zero values for winds, waves and currents. For example, in the case of having a 5-day wind forecast, but only a 3-day current forecast, it is still possible to run a 5-day trajectory forecast, where the current will be zero for the last 2 days.

A key feature of OpenDrift, for both convenience and robustness, is the possibility to provide a priority list of Readers for a given set of variables. As an example, it is possible to specify that a high-resolution ocean model shall be used whenever particles are within coverage in space and time, and reverting to using another model with larger coverage in space and time whenever particles are outside the time or spatial domain of the high-resolution model. As an important feature for operational setups, the backup Readers will also be used if the first-choice model (file or URL) should not be available, or if there should be any other problems, e.g. corrupt values or files.

\subsubsection{Seeding of elements}

The seeding methods of OpenDrift are very flexible. The simplest case is to seed (initialise) an element at a given position and time:

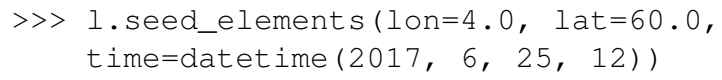

Also the number of elements and an uncertainty radius may be provided. Further, both position and time may be provided as two-element vectors to seed elements continuously in space and time from position $\mathrm{P} 1$ with uncertainty radius $\mathrm{R} 1$ at time $\mathrm{T} 1$, to position $\mathrm{P} 2$ with uncertainty radius $\mathrm{R} 2$ at time T2. This is a common use case in search-and-rescue modelling (see Breivik and Allen, 2008): a ship is known to have departed from position P1 at time T1, with normally small uncertainty radius $\mathrm{R} 1$, and disappeared on the way towards the destination (P2), normally with larger uncertainty in position (R2) and estimated arrival time (T2). Thus, this will track out a "seeding cone" in space and time. Another common use case is that P1 equals P2, with T2 $>\mathrm{T} 1$, e.g. simulating a continuous oil spill from a leaking well.

Another built-in feature is seeding of elements within polygons. This may, e.g. be done by providing vectors of longitude and latitude:

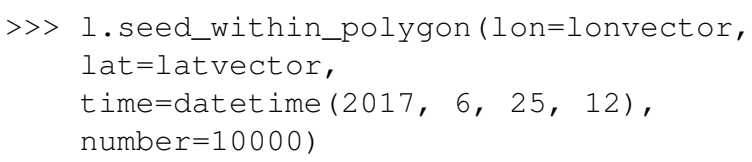

This example will seed 10000 elements with regular spacing within the polygon encompassed by vectors lonvector and latvector. Based upon this generic polygon seeding method, more specific applications have been developed; see, e.g. Sect. 3.2. The seeding methods may also be overloaded to provide customised functionality for a given module.

\subsubsection{Configuration}

OpenDrift modules share several configuration settings which may be adjusted before a simulation, as well as some settings which are module specific. All possible settings of a module may be shown with the command l.list_configspec (), of which one example is

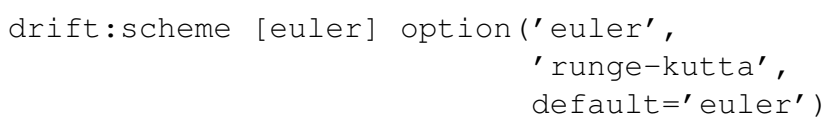

This shows that the setting drift: scheme may have one or two possible values, 'euler' or 'runge-kutta', where the first is the default and also the present setting as indicated within brackets. A second-order Runge-Kutta propagation scheme may instead be activated by the command

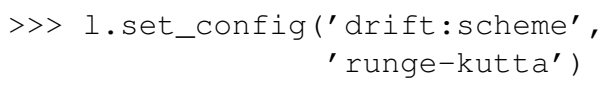

Another example of a configuration setting is coastline_action, which determines how the particles shall interact with the coastline. Possible options are stranding, which means that particles will be deactivated if they hit the coastline (default); previous which means that particles shall be moved back to their previous position (i.e. "waiting" at the coast until eventually moved offshore later); or none, which means that particles do not interact 
with land, as for the WindBlow module as demonstrated in Sect. 3.3.

The configuration mechanism is based on the widely used ConfigObj package and allows, e.g. exporting to, and importing from, files of the common 'INI' format.

\subsubsection{Starting the model run}

After initialisation, configuration, adding of Readers and seeding of elements, the model simulation may be started by calling the method run:

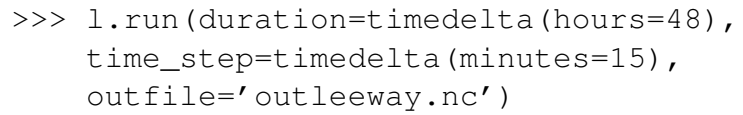

This starts the main loop, as shown on the flowchart of Fig. 1. At each time step, forcing data are obtained by all the Readers and interpolated onto the element positions, and the model-specific update method is called to move and/or otherwise update the other element properties (e.g. evaporation of oil elements, or growth of fish larvae) based on the environmental conditions.

For the above example, the simulation will cover $48 \mathrm{~h}$, starting the time of the first seeded elements. The time step of the calculation is given here as $15 \mathrm{~min}$. An output time step might be specified differently, with, e.g. output every hour to save memory and disk space.

All instances of OpenDrift can be run in reverse, i.e. backwards from a final destination, by reversing the sign of the advective increment. All spatial increments due to model physics pertinent to the instance in question are calculated as normal, but the sign of the total increment, $(\Delta x, \Delta y, \Delta z)$, is reversed and the particles are advected "backwards" over a time step $\Delta t$. All diffusive properties are kept in the forward sense, meaning that particles will disperse as they propagate backwards in time. Nonlinear processes, such as evaporation of oil or capsizing of vessels, are disabled in backtracking mode. This simple backtracking scheme is an easy-to-use alternative to more complicated inverse methods, such as iterative forward trajectory modelling (Breivik et al., 2012b), and is also much less computationally expensive.

\subsubsection{Exporting model output}

In the above example, the output is saved to a CF-compliant NetCDF file (trajectory data specification), which is the default output format of OpenDrift. Both particle positions and any other properties, as well as configuration settings are stored in the file. If the number of elements and time steps is too large to keep all data in physical memory, OpenDrift will flush history data to the output file as needed during the simulation to free internal memory. The simulation may be imported by OpenDrift, or independent software, for subsequent analysis or plotting. Stored output files may also be used as input to a subsequent OpenDrift simulation, allowing for an intermediate step where the particles are subjected to various considerations such as a Bayesian update of their probabilities based on posterior information. Saving data to files is not a requirement, as the output of the simulations is otherwise held in memory for subsequent plotting or analysis, either interactively from within Python shell, or by a script. A number of visualisation tools based on the Matplotlib graphics library of Python are included within OpenDrift. Some examples of both generic and module-specific plotting methods are illustrated in Sect. 3 .

\section{Examples of model instances}

\subsection{Leeway (search and rescue)}

The OpenDrift Leeway instance (OpenLeeway) is based on the operational search-and-rescue model of the Norwegian Meteorological Institute (Breivik and Allen, 2008). The model ingests a list of object classes, where each drifting object has specific properties such as downwind and crosswind leeway (the motion due to wind) in a way similar to SAROPS, the operational system used by the US Coast Guard (see Kratzke et al., 2010, and the overview of searchand-rescue models by Davidson et al., 2009). These properties vary greatly from object to object and are based on field work (Breivik et al., 2011, 2012a) where specific objects of relevance in search and rescue have been studied. All objects are assumed to be small enough that direct wave scattering forces are insignificant. Furthermore, the Stokes drift (Kenyon, 1969; Breivik et al., 2014, 2016) is inherently part of the leeway obtained from observations. As wind-generated waves have a mean direction closely aligned with the local wind direction, it is neither practical nor desirable to disentangle the Stokes drift from the wind drag for Leeway simulations.

Once an object class has been chosen and the pertinent wind and current forcing fields selected, the particles are seeded based on the available information. If the particles hit the coast, they stick by default. This can, however, be relaxed so that particles detach from the coastline if the wind direction changes.

The OpenLeeway class along with all other subclasses has the option of being run backwards. This is a convenient feature in the cases where, for example, a debris field is observed and the location of the accident is sought. Note that this method is fundamentally different from the BAKTRAK model described by Breivik et al. (2012b) where a large number of particles were seeded in potential initial locations at various times, and only those that ended up close to the location of the observed object were kept. This is an iterative procedure which in principle can deal with nonlinearities in the flow field as well as nonlinear behaviour of the object itself (such as capsizing and swamping). Although in principle this allows for a more realistic mapping of initial locations, the difficulties associated with this iterative process 
mean that real-time operations are normally better off with a simple negative-time integration.

OpenLeeway is used operationally at Norwegian Meteorological Institute and is also currently being implemented as the operational search-and-rescue model for the Joint Rescue Coordination Centres (JRCC) of Norway.

The following lines of Python code illustrate a complete working example of running an OpenLeeway simulation:

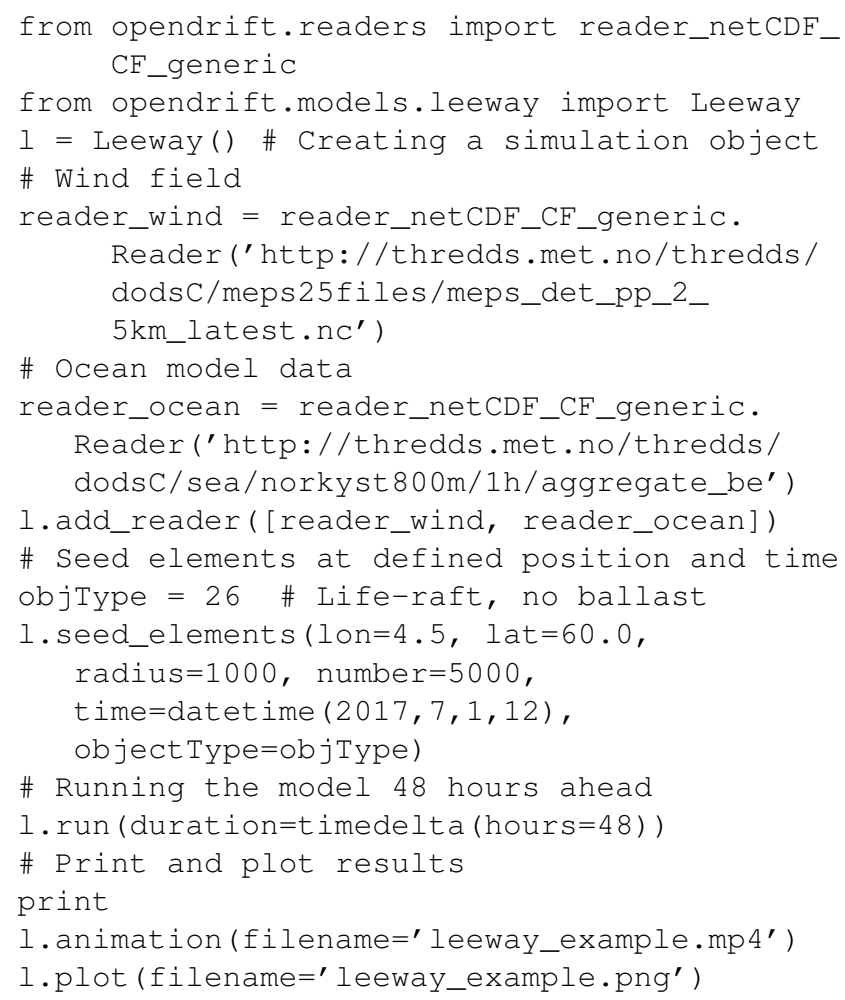

The final plotting command yields Fig. 2. The coastline shown is from the GSHHS database (Wessel and Smith, 1996), which is the default option used to check stranding in OpenDrift. This coastline is, however, interfaced to OpenDrift as a regular Reader (Sect. 2.1) and can be replaced by any other Reader providing the CF variable land_binary_mask. This allows performing simulations in narrow bays or lakes where even the full-resolution GSHHS coastline is too coarse.

\subsection{OpenOil (oil drift)}

OpenOil is a full-fledged oil drift model, bundled within the OpenDrift framework. As a model, it has been developed from scratch but is based on a selection of parameterisations of oil drift as found in the open research literature. With regard to horizontal drift, three processes are considered:

- Any element, whether submerged or at the surface, drifts along with the ocean current.

- Elements are subject to Stokes drift corresponding to their actual depth. Surface Stokes drift is normally obtained from a wave model (or by any Reader), and its decline with depth is calculated as described in Breivik et al. (2016).

- Oil elements at the ocean surface are moved with an additional factor of $2 \%$ (configurable) of the wind. Together with the Stokes drift (typically $1.5 \%$ of the wind at the surface), this sums up to the commonly found empirical value of $3.5 \%$ of the wind (Schwartzberg, 1971). The physical mechanism behind this wind drift factor is not obvious and is discussed in Jones et al. (2016).

The above three drift components may lead to a very strong gradient of drift magnitude and direction in the upper few metres of the ocean. For this reason, it is also of critical importance to have a good description of the vertical oil transport processes, which in OpenOil are the sum of the following factors:

- If the vertical ocean current velocity is available from a Reader, the oil elements will follow it. This part of the movement is, however, often negligible compared to the processes below.

- Oil elements at the surface, regarded as being in the state of an oil slick, may be entrained into the ocean by breaking waves. Presently, OpenOil contains two different parameterisations of this entrainment rate, from which the user can chose as part of configuration (see below): Tkalich and Chan (2002) and Li et al. (2017). The entrainment depends on both the wind and wave (breaking) conditions and also on the oil properties, such as viscosity, density and oil-water interfacial tension.

- Buoyancy of droplets is calculated according to empirical relationships and the Stokes law of Tkalich and Chan (2002), dependent on ocean stratification (calculated from temperature and salinity profile, normally read from an ocean model), oil and water viscosities and densities. Also, the buoyancy is strongly dependent on the oil droplet size (diameter), of which two parameterisations are available: one is a generic power law, with droplets between a minimum and maximum diameter, and a configurable exponent where -2.3 corresponds to the classical work of (Delvigne and Sweeney, 1989). The second option for droplet size spectrum is a "modern" approach by Johansen et al. (2015), where a lognormal droplet spectrum is calculated explicitly based on wave height and oil properties such as viscosity, density, interfacial tension and surface film thickness.

- In addition to the wave-induced entrainment, the oil elements are also subject to vertical turbulence throughout the water column, as parameterised with a numerical scheme described in Visser (1997). This scheme is generic within OpenDrift and is also used by the PelagicEgg module for ichthyoplankton (Sect. 3.4). Only the properties specific to oil, or plankton, are coded in the respective classes (modules). 


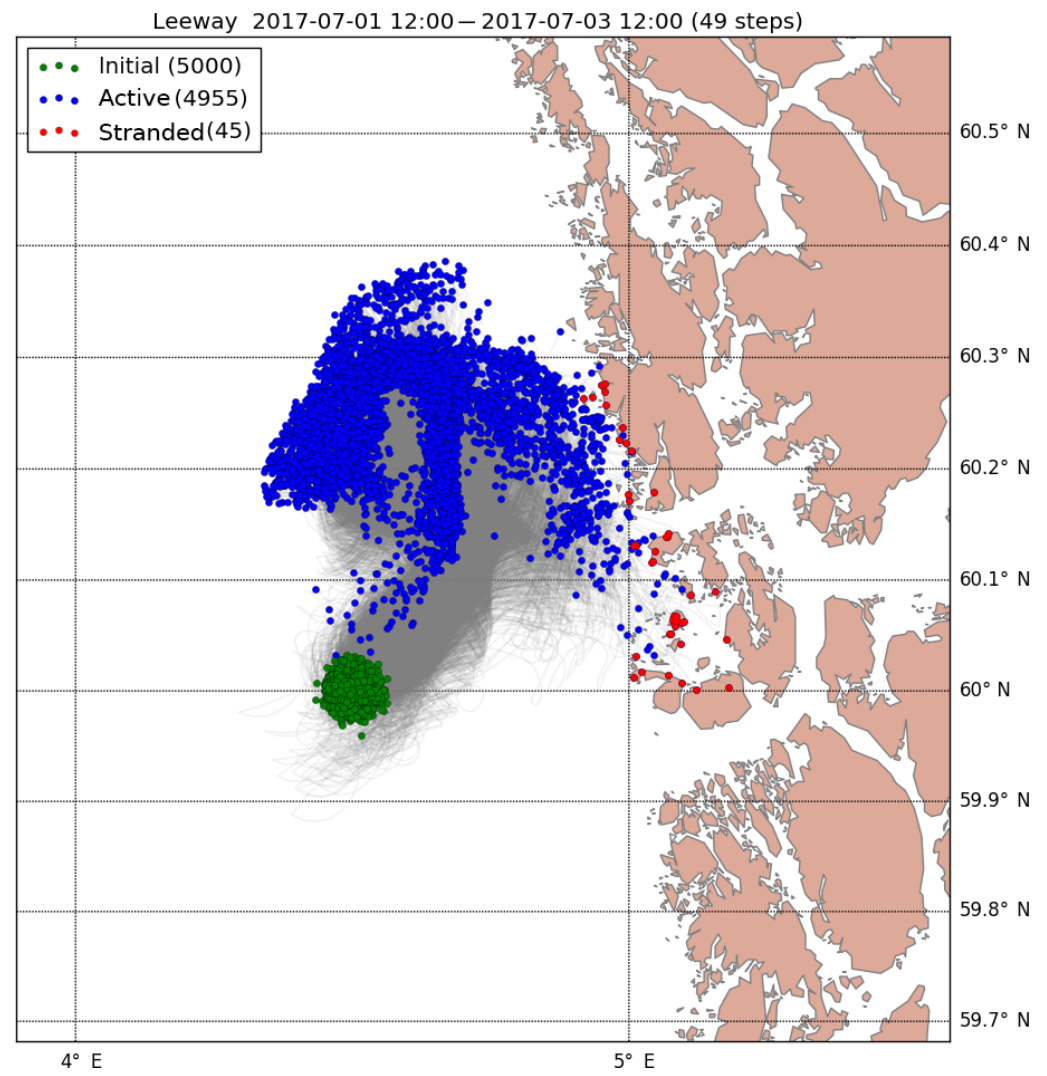

Figure 2. Output from the Leeway example of Sect. 3.1. Green dots are the initial positions of the elements (life rafts), grey lines are trajectories, and blue dots are positions at the end of the simulation. Red dots indicate elements which have hit land (stranded).

In addition to the vertical and horizontal drift, weathering of the oil also has to be considered. While parameterisations of weathering might also be implemented directly within the OpenDrift framework, the OpenOil module instead interfaces with the already existing OilLibrary software developed by NOAA (https://github.com/NOAA-ORR-ERD/OilLibrary). The NOAA OilLibrary is also open-source and written in Python, so integration is straightforward. In addition to state-of-the-art parameterisations of processes such as evaporation, emulsification and dispersion, this software contains a database of measured properties of almost 1000 oil types from around the world. As oils from different sources/wells have vastly different properties, such a database is of vital importance for accurate results. The same OilLibrary is also used by the NOAA oil drift model PyGNOME (https://github.com/NOAA-ORR-ERD/PyGnome), where it is replacing the original ADIOS oil library (Lehr et al., 2002). PyGNOME includes also more processes not (yet) included in OpenOil, such as dissolution, and adding of dispersants.

To run an OpenOil simulation, one could reuse the exact code as for the Leeway example of Sect. 3.1, only replacing the name of the imported module (OpenOil instead of Leeway) and replacing the objectType property of Leeway with a corresponding oil name from the NOAA database. However, whereas key features and functionality is shared among OpenDrift modules, each module (or group of modules) may add specific functionality. For example, for OpenOil, it is possible to initialise the simulations with an oil slick as read from a file containing contours, either a shapefile or the $\mathrm{Ge}$ ography Markup Language (GML) format/specification, as used by the European Maritime Safety Agency (EMSA):

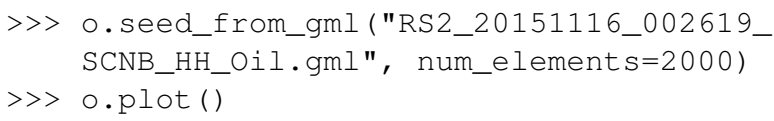

where o is the oil drift simulation object. The last command produces the plot shown in Fig. 3 .

OpenOil also has module-specific configuration settings. The following commands specify that the oil entrainment rate shall be calculated according to $\mathrm{Li}$ et al. (2017), and the oil droplet size spectrum shall be calculated according to Johansen et al. (2015).

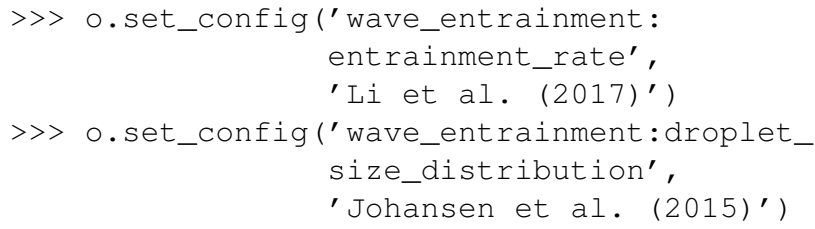




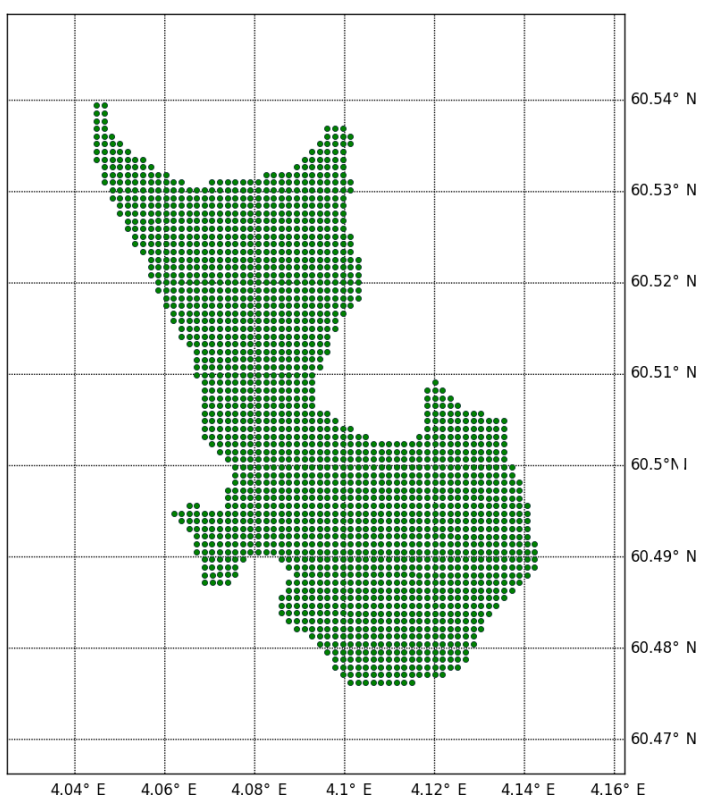

Figure 3. Oil drift simulation initialised by seeding 2000 oil elements within contours of an oil slick as observed from satellite (Radarsat2). The contour is imported from a Geography Markup Language (GML) file produced by Kongsberg Satellite Services (KSAT).

Adjusting configuration this way is convenient for sensitivity studies, where one component is changed for two otherwise identical simulations.

After the simulation is finished, the generic plot command may be used to produce a map with trajectories as shown in Fig. 2. However, more specific plotting methods are also available. The command $\circ . p l o t \_o i l \_b u d g e t()$ plots an oil budget as shown in Fig. 4.

The vertical distribution of the elements can be plotted with the command o.plot_vertical_distribution(), generating output as shown in Fig. 5. This method is shared among three-dimensional modules and may also be used for simulations with, e.g. the PelagicEgg module (Sect. 3.4).

\subsection{WindBlow (atmospheric transport)}

As an example of a minimalistic trajectory model, we also include the instance WindBlow, which simply calculates the propagation of a passive particle subject to a twodimensional wind field. The code below is the complete and fully functional WindDrift module.

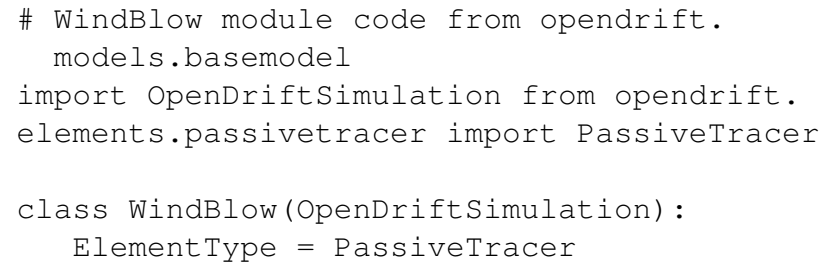

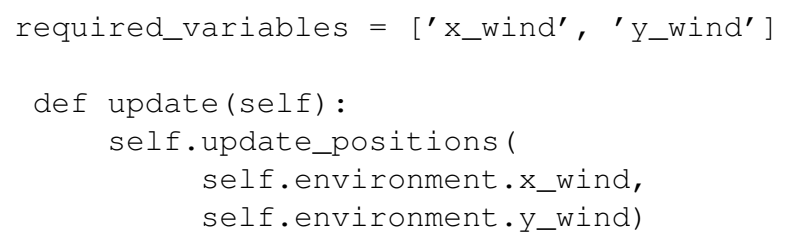

Because all common functionality is inherited from the main class, the WindBlow model only needs to address its own specific needs. It will use elements without any other properties except for position (PassiveTracer), and the only forcing needed to move the elements is wind, whose vector components are named $x \_$wind and $y \_$wind in CF terminology. The update() method which is called at each time step simply advects all elements with the wind velocity at their respective locations. The wind might be provided by any Reader (Sect. 2.1). The WindDrift module may be run with an even more simplified form of the code example found in Sect. 3.1: the WindDrift class has to be imported, no Reader for ocean current is needed, and there is no object category to specify.

Clearly, an air parcel in the real atmosphere will also be subjected to updrafts and diffusion, and will with time rise or fall, but the example serves to demonstrate how little is required to develop a new subclass of OpenDrift. The model may be made more sophisticated by adding, e.g. vertical wind (upward_air_velocity) and turbulence parameters to the list of required variables, and adding corresponding parameterisations of how to use this information for the advection.

\subsection{Other modules}

In addition to the models described above, some other modules are bundled within the OpenDrift repository, as illustrated in Fig. 6:

- OceanDrift is a basic module for tracking, e.g. water masses or passive tracers. Stokes drift is included, if provided by a Reader. A wind drift factor may also be specified, allowing an additional wind drag at the surface, e.g. for simulation trajectories of various ocean drifting buoys (Dagestad and Röhrs, 2018).

- PelagicEgg is a module for transport of pelagic ichthyoplankton. This module contains quite basic functionality with identical transport mechanisms as in Röhrs et al. (2014), including the vertical turbulent scheme (Sect. 3.2) which is of key importance for most pelagic plankton applications. Although a fully working a module, users with specialised needs (e.g. a specific biological species) can customise the drift and behaviour parameterisations by modifying or adding parameterisations in the PelagicEgg module, such as larval behaviour. Some users have interfaced this module with existing Fortran code, e.g. for calculation of sunlightdependent behaviour; see, e.g. Kvile et al. (2018) and 


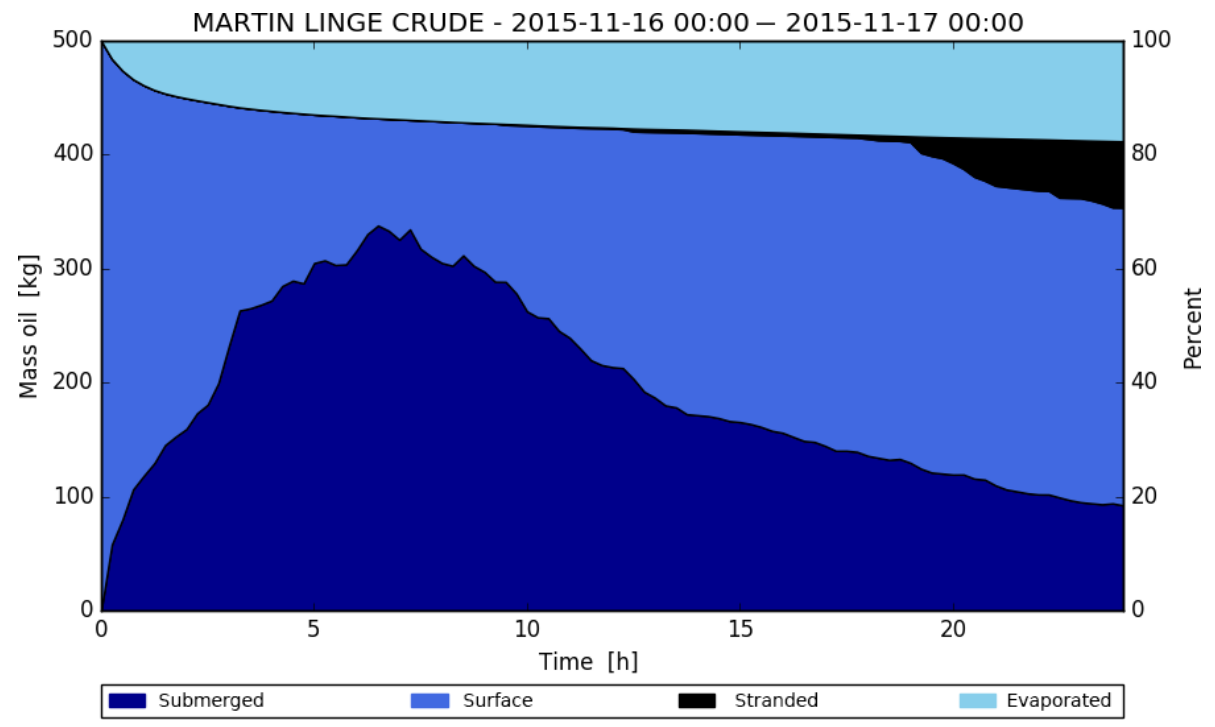

Figure 4. Plot of the time evolution of the oil budget of a $24 \mathrm{~h}$ simulation with OpenOil. Of $500 \mathrm{~kg}$ oil initially at the ocean surface, about $20 \%$ is seen to evaporate within the $24 \mathrm{~h}$. The amount of oil submerged due to wave action and ocean turbulence varies with the wind and wave conditions, with more oil resurfacing when wind decreases after about $7 \mathrm{~h}$. After about $18 \mathrm{~h}$, some of the oil is seen to hit the coastline. These results are for the oil type "Martin Linge crude"; very different results could be obtained if using another oil type for the same geophysical conditions.

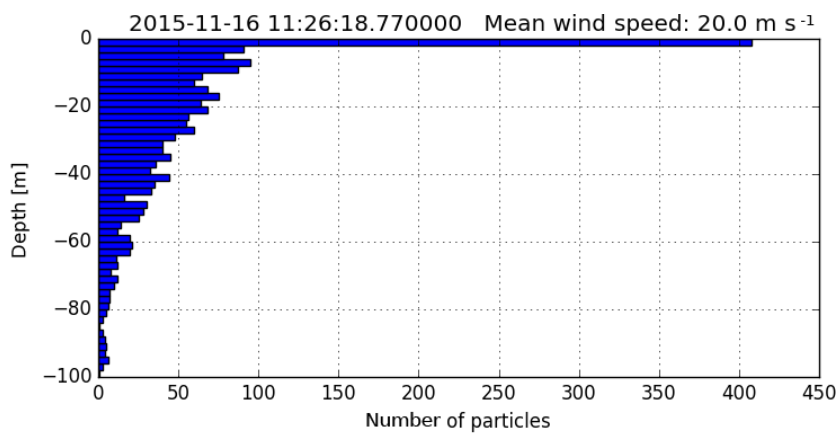

Time step

Figure 5. Vertical profile of the amount of (oil) elements. The bottom bar is an interactive slider, which the user can pull left/right to see the time variation of the vertical distribution.

Sundby et al. (2017). A pure Python version of the sunlight module is available and will be included in a future version of OpenDrift.

- ShipDrift is a module for predicting the drift of ships larger than $30 \mathrm{~m}$, where the effect of waves has to be calculated explicitly, and not implicitly with the wind drift as in the Leeway module. This module is based on Sørgård and Vada (2011). A previous version programmed in the $\mathrm{C}$ programming language has been used operationally at MET Norway for 15 years but is now replaced by the OpenDrift version, which has been tested and shown to provide identical output for identical input/forcing.

A module for drift of icebergs (OpenBerg, not yet included in repository) has been developed by Ron Saper at Carleton University with partial funding from ASL Environmental Sciences of Victoria, Canada, and with data support from the Canadian Ice Service (personal communication, 2017). Two different iceberg drift forecasting approaches are being tested. One approach uses a drag formulation to calculate wind and water drag forces. The challenge with this approach is that the trajectories are very sensitive to underwater draft/shape and suitable drag coefficients, of which information is rarely available. The second approach predicts and subtracts the wind and tidal components of the drift, and then analyses the residual for extrapolation an appropriately short time into the future. Finally, wind and tidal components are added back in to produce a trajectory forecast. The first version of OpenBerg does not include thermodynamic effects (melting) which are important longer timescales from weeks to months.

Drift of marine plastics, including microplastics, is an important application not covered by modules included with the OpenDrift repository version 1.0. However, as most of the needed infrastructure is already provided, including a vertical mixing scheme, a user with knowledge of the relevant physics and basic Python programming should be able to implement such a module with moderate efforts. However, there is no upper limit to the complexity of any module. 


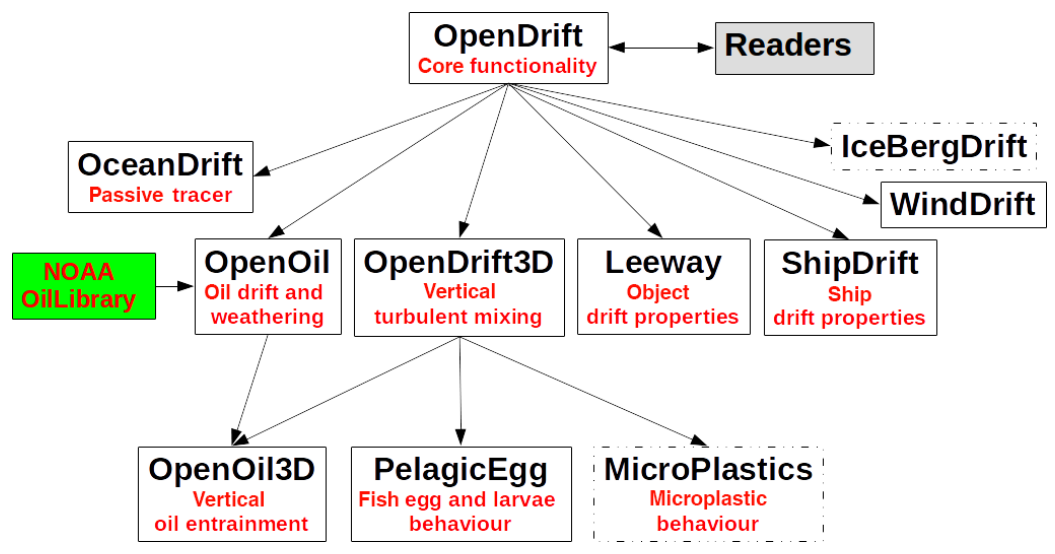

Figure 6. Illustration of how OpenDrift modules for specific applications (white boxes) inherit common functionality from the core module. This includes functionality to interact with Readers for obtaining forcing data. Subclassing (inheritance) allows, e.g. both the OpenOil and PelagicEgg models to share further 3-D-functionality through subclassing the OpenDrift3D class. The boxes with solid boundaries illustrate existing modules bundled with the OpenDrift repository, whereas dashed boundaries indicate planned modules. The green box illustrates that OpenOil (oil drift model) utilises functionality from a third-party library, the NOAA OilLibrary.

\section{Test suite and example scripts}

OpenDrift contains a broad set of automatic tests (Python unittest framework) which can be run by the user to assure that all calculations are performed correctly on the local machine. The tests cover both basic calculations, such as interpolation and rotation of vectors from one spatial reference system (SRS) to another, but also more extensive integration tests, performing full simulations with the modules to verify that an expected numerical result is obtained. Also, very importantly, the tests are also run automatically on a variety of machine configurations, using the Travis Continuous Integration (CI) framework (https://travis-ci.org). This ensures that OpenDrift calculations remain accurate and correct with both old and new versions of the various required libraries (e.g. NumPy), and that existing functionality is not broken as new functionality is added. For version 1.0 of OpenDrift, $64 \%$ of the code is covered by the unit tests, as reported by the Coveralls tool (coveralls.io).

A user manual of OpenDrift is kept alongside the code repository on the wiki pages of GitHub (https://github.com/ OpenDrift/opendrift/wiki), facilitating a dynamic description to evolve with the code, instead of diverting from it. Many example scripts (40 in version 1.0) are also provided in the repository along with the needed input forcing data, illustrating a variety of real-life use cases. The examples can easily be modified and adapted, allowing a soft learning curve.

OpenDrift also comes with a set of handy command line tools, such as readerinfo.py, which may be used to easily inspect the contents and coverage of potential forcing fields. The following shell command produces the same output as the example of Sect. 2.1, where the switch '-p' also displays a plot of the geographical coverage:

\$ readerinfo.py ocean_model_output.nc -p

\section{Graphical user interfaces}

Although running OpenDrift modules with Python scripts (see, e.g. Sect. 3.1) is the most flexible and powerful, a basic graphical user interface (GUI) is also included in the repository. A screenshot is shown in Fig. 7. The GUI allows to select a module and an object type or medium (e.g. oil type) corresponding to the module, and then a seeding location and time. The simulation is started by clicking the "START" button, and plots and animation of the output is available after the simulation and also saved to a NetCDF file. The GUI will obtain forcing data through a provided list (configurable) of Thredds servers with global coverage, so there is no need for the user to obtain and download large amounts of model input in advance. Although presently with only basic functionality, the GUI is in operational use at MET Norway, where it is tested daily by meteorologists on duty as part of the oil spill and search-and-rescue preparedness system.

In addition to the native GUI, a web interface has also been developed for remote access without need for any local installation. This is based on communication with OpenDrift through a web processing service (WPS) developed at MET Norway (not included in the repository). Independently, a WPS for the Leeway module has also been developed and implemented at the Swedish Met Office (SMHI). A generic and configurable WPS to be included in the repository is planned for the future.

\section{Discussion and conclusions}

Several offline trajectory models exist to predict the transport and transformation of various substances and objects in the ocean or in the atmosphere. OpenDrift is an open-source Python framework aiming at extracting anything which is 


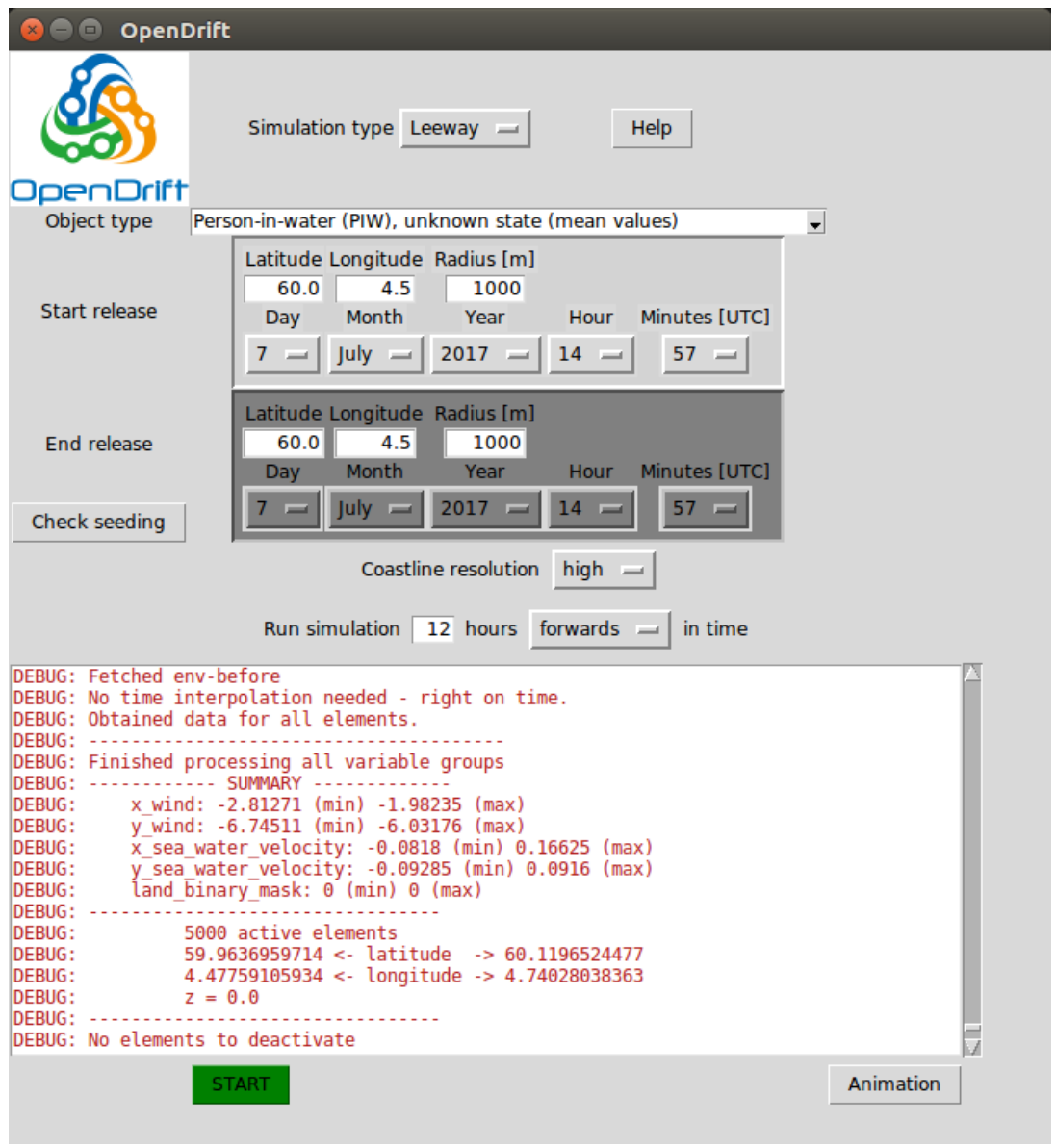

Figure 7. Screenshot of the graphical user interface included with OpenDrift.

common to all such trajectory models in a core library, and combining this with a clean and generic interface for describing any processes which are application specific. Several examples of such specific modules are bundled with the OpenDrift code repository and serve as ready-to-use trajectory models. This includes an oil drift model (OpenOil), a search-and-rescue model (Leeway) and a model for predicting the drift and transformation of ichthyoplankton (PelagicEgg). Interfaces (Readers) towards the most common formats of forcing data (e.g. NetCDF and GRIB) are also included, allowing any of the modules to be forced by data from a combination of files and other sources, including remote Thredds servers. The concept of Readers is also modularised, allowing a scientist or programmer to easily develop an interface towards any other specific source of forcing data, e.g. an ASCII file containing in situ observations from a buoy or weather station, or ocean currents from HF-radar systems in a specific binary format.

A built-in configuration mechanism provides flexibility to the operation of the OpenDrift modules. However, the fact that the application-specific processes of these modules are separated from the technical complexities of the OpenDrift core provides even greater flexibility to the user in that it is easy to modify existing modules, or even write new modules from scratch. Several users have already developed or adjusted modules for their specific purpose and added useful contributions to the OpenDrift core (Sundby et al., 2017; Kvile et al., 2018).

Whereas flexibility is important for scientific studies, OpenDrift is also designed for performance and robustness and is in daily use for operational emergency response systems at the Norwegian Meteorological Institute. Being able to use the same tool in both cases facilitates rapid transition of the latest research results into operations.

The efficiency of the code has been optimised to the point that more time is normally spent on reading the forcing data from disk (or a URL) than on performing actual calculations. Computational performance similar to compiled languages (Fortran or C) is obtained by, e.g. using primarily NumPy arrays for calculations (avoiding the slower MaskedArray class) and avoiding "for loops". A typical emergency simulation with the Leeway model with 5000 elements and $48 \mathrm{~h}$ duration takes on the order of $1 \mathrm{~min}$. A corresponding simulation with OpenOil takes about 3-5 min, primarily because 
more layers of ocean model data have to be read from disk, in contrast to the Leeway simulation which only needs the surface current. A typical 1-year simulation of 20000 drifting cod eggs (developing into larvae) takes about $4 \mathrm{~h}$ on a regular desktop computer (Kvile et al., 2018). The OpenDrift code is presently not parallelised. However, given that most time is spent on reading data from disk, some further performance gain could possibly be achieved by, e.g. reading in parallel data from different input files (e.g. ocean model and atmospheric model) or by reading input data for the following time step in parallel to performing calculations.

Another great benefit of the modularity provided by OpenDrift is the ability to perform sensitivity tests by varying one component while keeping everything else constant. Much can be learnt from performing two otherwise identical simulations with, e.g. input from two different Eulerian models, or by using two different parameterisations of some process. Further, consistency is also provided by the possibility of handling, e.g. overlap of fish eggs and oil with the same forcing and numerical scheme. Traditionally, it might be difficult to draw conclusions by comparing the output from different trajectory models, as the differences depend on many factors, such as interpolation schemes and numerical algorithms.

The modules presently included with OpenDrift will be improved in the future, in particular by validation against available relevant observations. Among the general problems which require more attention, is properly describing and quantifying the very strong vertical gradients of horizontal drift often found in the upper few metres of the ocean, as result of a delicate balance between ocean currents and Stokes drift, as well as the direct wind drift affecting objects and substances at the very ocean surface. This vertical gradient of forcing is highly important for drift of, e.g. oil and chemicals, plankton and microplastics. This implies further that having accurate parameterisations of the vertical transport processes (wave entrainment, buoyancy and ocean turbulence) is also very important. For example, a key factor for successful simulation of the drift of observed oil slicks in Jones et al. (2016) was to incorporate a vertical mixing scheme developed for fish eggs (Sundby, 1983; Thygesen and Ådlandsvik, 2007; Ådlandsvik and Sundby, 1994) into the oil drift model OpenOil.

Code availability. OpenDrift is housed on GitHub: https://github.com/OpenDrift/opendrift. The accompanying wiki pages contain installation instructions, documentation and examples. Version 1.0 of OpenDrift is registered with Zenodo: https://doi.org/10.5281/zenodo.845813. OpenDrift has been tested on both Linux, Mac and Windows platforms. Version 1.0 requires Python 2.7 and is not adapted for Python 3. The OpenDrift framework is distributed under a GPLv2 license.
Acknowledgements. Knut-Frode Dagestad, Johannes Röhrs and Øyvind Breivik gratefully acknowledge support from the Joint Rescue Coordination Centres through the project OpenLeeway and the Research Council of Norway through the CIRFA (grant no. 237906) and RETROSPECT (grant no. 244262) projects. The Norwegian Clean Seas Association (NOFO) and the Norwegian Coastal Administration have been instrumental in their support and testing of the software during the development phase.

Edited by: Robert Marsh

Reviewed by: two anonymous referees

\section{References}

Ådlandsvik, B. and Sundby, S.: Modelling the transport of cod larvae from the Lofoten area, ICES J. Mar. Sci. Symp., 198, 379392, available at: https://www.scienceopen.com/document?vid= 53b16ebf-bddc-4332-8719-1bb180e1cdaa (last access: 9 April 2018), 1994.

Allen, A. and Plourde, J. V.: Review of Leeway: Field Experiments and Implementation, US Coast Guard Research and Development Center, 1082 Shennecossett Road, Groton, CT, USA, Tech. Rep. CG-D-08-99, available through: http://www.ntis.gov (last access: 9 April 2018), 1999.

Bartnicki, J., Amundsen, I., Brown, J., Hosseini, A., Hov, O., Haakenstad, H., Klein, H., Lind, O. C., Salbu, B., Szacinski Wendel, C. C., and Ytre-Eide, M. A.: Atmospheric transport of radioactive debris to Norway in case of a hypothetical accident related to the recovery of the Russian submarine K-27, J. Environ. Radioactiv., 151, 404-416, https://doi.org/10.1016/j.jenvrad.2015.02.025, 2016.

Berry, A., Dabrowski, T., and Lyons, K.: The oil spill model OILTRANS and its application to the Celtic Sea, Mar. Pollut. Bull., 64, 2489-2501, https://doi.org/10.1016/j.marpolbul.2012.07.036, 2012.

Blanke, B., Raynaud, S., Blanke, B., and Raynaud, S.: Kinematics of the Pacific Equatorial Undercurrent: An Eulerian and Lagrangian Approach from GCM Results, J. Phys. Oceanogr., 27, 1038-1053, https://doi.org/10.1175/15200485(1997)027<1038:KOTPEU>2.0.CO;2, 1997.

Breivik, Ø. and Allen, A. A.: An operational search and rescue model for the Norwegian Sea and the North Sea, J. Marine Syst., 69, 99-113, https://doi.org/10.1016/j.jmarsys.2007.02.010, 2008.

Breivik, Ø., Allen, A. A., Maisondieu, C., and Roth, J. C.: Wind-induced drift of objects at sea: The leeway field method, Appl. Ocean Res., 33, 100-109, https://doi.org/10.1016/j.apor.2011.01.005, 2011.

Breivik, Ø., Allen, A., Maisondieu, C., Roth, J.-C., and Forest, B.: The Leeway of Shipping Containers at Different Immersion Levels, Ocean Dynam., 62, 741-752, https://doi.org/10.1007/s10236-012-0522-z, 2012a.

Breivik, Ø., Bekkvik, T. C., Ommundsen, A., and Wettre, C.: BAKTRAK: Backtracking drifting objects using an iterative algorithm with a forward trajectory model, Ocean Dynam., 62, 239-252, https://doi.org/10.1007/s10236-011-0496-2, 2012b. 
Breivik, Ø., Allen, A., Maisondieu, C., and Olagnon, M.: Advances in Search and Rescue at Sea, Ocean Dynam., 63, 83-88, https://doi.org/10.1007/s10236-012-0581-1, 2013.

Breivik, Ø., Janssen, P., and Bidlot, J.: Approximate Stokes Drift Profiles in Deep Water, J. Phys. Oceanogr., 44, 2433-2445, https://doi.org/10.1175/JPO-D-14-0020.1, 2014.

Breivik, Ø., Bidlot, J.-R., and Janssen, P. A.: A Stokes drift approximation based on the Phillips spectrum, Ocean Model., 100, 4956, https://doi.org/10.1016/j.ocemod.2016.01.005, 2016.

Brovchenko, I., Kuschan, A., Maderich, V., Shliakhtun, M., Yuschenko, S., and Zheleznyak, M.: The modelling system for simulation of the oil spills in the Black Sea, Elsev. Oceanogr. Serie., 69, 586-591, https://doi.org/10.1016/S04229894(03)80095-8, 2003.

Dagestad, K.-F. and Röhrs, J.: Prediction of ocean surface trajectories using satellite derived vs. modeled ocean currents, Remote Sens. Environ., submitted, 2018.

Dagestad, K.-F., Röhrs, J., Breivik, Ø., and Aadlandsvik, B.: OpenDrift v1.0.3, https://doi.org/10.5281/zenodo.1175634, 2017.

Daniel, P.: Operational forecasting of oil spill drift at Météo-France, Spill Sci. Technol. B., 3, 53-64, https://doi.org/10.1016/S13532561(96)00030-8, 1996.

Davidson, F. J. M., Allen, A., Brassington, G. B., Breivik, Ø., Daniel, P., Kamachi, M., Sato, S., King, B., Lefevre, F., Sutton, M., and Kaneko, H.: Applications of GODAE ocean current forecasts to search and rescue and ship routing, Oceanography, 22, 176-181, https://doi.org/10.5670/oceanog.2009.76, 2009.

De Dominicis, M., Pinardi, N., Zodiatis, G., and Lardner, R.: MEDSLIK-II, a Lagrangian marine surface oil spill model for short-term forecasting - Part 1: Theory, Geosci. Model Dev., 6, 1851-1869, https://doi.org/10.5194/gmd-6-1851-2013, 2013.

Delvigne, G. and Sweeney, C.: Natural Dispersion of Oil, Publication No. 399, Delft Hydraulic Laboratory, Delft, The Netherlands, Tech. rep., 399, 30 pp., 1989.

Dick, S. and Soetje, K. C.: An operational oil dispersion model for the German Bight, Hydrographische Zeitschrift, 16, 43 pp., 1990.

Döös, K., Kjellsson, J., and Jönsson, B.: TRACMASS - A Lagrangian Trajectory Model, in: Preventive Methods for Coastal Protection, Springer International Publishing, Heidelberg, 225249, https://doi.org/10.1007/978-3-319-00440-2_7, 2013.

Johansen, O., Reed, M., and Bodsberg, N. R.: Natural dispersion revisited, Mar. Pollut. Bull., 93, 20-26, https://doi.org/10.1016/j.marpolbul.2015.02.026, 2015.

Jones, C. E., Dagestad, K.-F., Breivik, O., Holt, B., Röhrs, J., Christensen, K. H., Espeseth, M., Brekke, C., and Skrunes, S.: Measurement and modeling of oil slick transport, J. Geophys. Res.-Oceans, 121, 7759-7775, https://doi.org/10.1002/2016JC012113, 2016.

Kenyon, K. E.: Stokes Drift for Random Gravity Waves, J. Geophys. Res., 74, 6991-6994, https://doi.org/10.1029/JC074i028p06991, 1969.

Kratzke, T. M., Stone, L. D., and Frost, J. R.: Search and Rescue Optimal Planning System, in: Information Fusion (FUSION), 2010 13th Conference on, 26-29 July 2010, Edinburgh, UK, IEEE, 8 pp., https://doi.org/10.1109/ICIF.2010.5712114, 2010.

Kubat, I., Sayed, M., Savage, S. B., Carrieres, T., and Crocker, G.: An Operational Iceberg Deterioration Model, in: The Seventeenth International Offshore and Polar Engineering Conference, 1-6 July, Lisbon, Portugal, International Society of Offshore and Polar Engineers, available at: https://www.onepetro.org/ conference-paper/ISOPE-I-07-282 (last access: 9 April 2018), 2007.

Kvile, K., Romagnoni, G., Dagestad, K.-F., Langangen, Ø. and Kristiansen, T.: Sensitivity of modelled North Sea cod larvae transport to vertical behaviour, ocean model resolution and interannual variation in ocean dynamics, ICES Journal of Marine Science, in press, 2018.

Lardner, R., Zodiatis, G., Loizides, L., and Demetropoulos, A.: An operational oil spill model for the Levantine Basin (Eastern Mediterranean Sea), International symposium on marine pollution, Monaco, Monaco, 5-9 October 1998, 30, 511-512, 1998.

Lehr, W., Jones, R., Evans, M., Simecek-Beatty, D., and Overstreet, R.: Revisions of the ADIOS oil spill model, Environ. Modell. Softw., 17, 189-197, https://doi.org/10.1016/S13648152(01)00064-0, 2002.

Li, Z., Spaulding, M. L., and French-McCay, D.: An algorithm for modeling entrainment and naturally and chemically dispersed oil droplet size distribution under surface breaking wave conditions, Mar. Pollut. Bull., 119, 145-152, https://doi.org/10.1016/j.marpolbul.2017.03.048, 2017.

Margvelashvily, N., Maderich, V., and Zheleznyak, M.: THREETOX - A Computer Code to Simulate Three-Dimensional Dispersion of Radionuclides in Stratified Water Bodies, Radiat. Prot. Dosim., 73, 177-180, 1997.

McKenna, D. S., Konopka, P., Grooß, J., Günther, G., Müller, R., Spang, R., Offermann, D., and Orsolini, Y.: A new Chemical Lagrangian Model of the Stratosphere (CLaMS) 1. Formulation of advection and mixing, J. Geophys. Res., 107, 4309, https://doi.org/10.1029/2000JD000114, 2002.

Paris, C. B., Helgers, J., van Sebille, E., and Srinivasan, A.: Connectivity Modeling System: A probabilistic modeling tool for the multi-scale tracking of biotic and abiotic variability in the ocean, Environ. Modell. Softw., 42, 47-54, https://doi.org/10.1016/J.ENVSOFT.2012.12.006, 2013.

Röhrs, J., Christensen, K. H., Vikebø, F., Sundby, S., Sætra, Ø., and Broström, G.: Wave-induced transport and vertical mixing of pelagic eggs and larvae, Limnol. Oceanogr., 59, 1213-1227, https://doi.org/10.4319/lo.2014.59.4.1213, 2014.

Schemm, S., Nummelin, A., Kvamstø, N. G., and Breivik, O.: The Ocean Version of the Lagrangian Analysis Tool LAGRANTO, J. Atmos. Ocean. Tech., 34, 1723-1741, https://doi.org/10.1175/JTECH-D-16-0198.1, 2017.

Schlag, Z. R. and North, E. W.: Lagrangian TRANSport model (LTRANS v.2) User's Guide, University of Maryland Center for Environmental Science, Horn Point Laboratory, Cambridge, MD, USA, Tech. rep., 183 pp., 2012.

Schwartzberg, H. G.: THE MOVEMENT OF OIL SPILLS, International Oil Spill Conference Proceedings, 1971, 489-494, https://doi.org/10.7901/2169-3358-1971-1-489, 1971.

Shchepetkin, A. F. and McWilliams, J. C.: The regional oceanic modeling system (ROMS): a split-explicit, free-surface, topography-following-coordinate oceanic model, Ocean Model., 9, 347-404, https://doi.org/10.1016/j.ocemod.2004.08.002, 2005.

Simpson, D., Benedictow, A., Berge, H., Bergström, R., Emberson, L. D., Fagerli, H., Flechard, C. R., Hayman, G. D., Gauss, M., Jonson, J. E., Jenkin, M. E., Nyíri, A., Richter, C., Semeena, V. S., Tsyro, S., Tuovinen, J.-P., Valdebenito, Á., 
and Wind, P.: The EMEP MSC-W chemical transport model - technical description, Atmos. Chem. Phys., 12, 7825-7865, https://doi.org/10.5194/acp-12-7825-2012, 2012.

Sørgård, E. and Vada, T.: Observations and modelling of drifting ships, DnV, Report 96-2011, Tech. rep., 64 pp., 2011.

Sprenger, M. and Wernli, H.: The LAGRANTO Lagrangian analysis tool - version 2.0, Geosci. Model Dev., 8, 2569-2586, https://doi.org/10.5194/gmd-8-2569-2015, 2015.

Stein, A. F., Draxler, R. R., Rolph, G. D., Stunder, B. J. B., Cohen, M. D., Ngan, F., Stein, A. F., Draxler, R. R., Rolph, G. D., Stunder, B. J. B., Cohen, M. D., and Ngan, F.: NOAA's HYSPLIT Atmospheric Transport and Dispersion Modeling System, B. Am. Meteorol. Soc., 96, 2059-2077, https://doi.org/10.1175/BAMSD-14-00110.1, 2015.

Stohl, A., Wotawa, G., Seibert, P., Kromp-Kolb, H., Stohl, A., Wotawa, G., Seibert, P., and Kromp-Kolb, H.: Interpolation Errors in Wind Fields as a Function of Spatial and Temporal Resolution and Their Impact on Different Types of Kinematic Trajectories, J. Applied Meteorol., 34, 2149-2165, https://doi.org/10.1175/15200450(1995)034<2149:IEIWFA>2.0.CO;2, 1995.

Sundby, S.: A one-dimensional model for the vertical distribution of pelagic fish eggs in the mixed layer, Deep-Sea Res. Pt. A, 30, 645-661, https://doi.org/10.1016/0198-0149(83)90042-0, 1983.

Sundby, S., Kristiansen, T., Nash, R., and Johannessen, T.: Dynamic Mapping of North Sea Spawning - Report of the KINO Project, Fisken og Havet, Intitute of Marine Research, Norway, Bergen, Tech. rep. 2-2017, 195 pp., available at: http://www.imr.no/filarkiv/2017/02/fogh_nr_2-2017_ kino_report_ss.pdf_1/nb-no (last access: 9 April 2018), 2017.

Thygesen, U. and Ådlandsvik, B.: Simulating vertical turbulent dispersal with finite volumes and binned random walks, Mar. Ecol. Prog. Ser., 347, 145-153, https://doi.org/10.3354/meps06975, 2007.
Tkalich, P. and Chan, E. S.: Vertical mixing of oil droplets by breaking waves, Mar. Pollut. Bull., 44, 1219-1229, https://doi.org/10.1016/S0025-326X(02)00178-9, 2002.

van Sebille, E., England, M. H., and Froyland, G.: Origin, dynamics and evolution of ocean garbage patches from observed surface drifters, Environ. Res. Lett., 7, 044040, https://doi.org/10.1088/1748-9326/7/4/044040, 2012.

van Sebille, E., Wilcox, C., Lebreton, L., Maximenko, N., Hardesty, B. D., van Franeker, J. A., Eriksen, M., Siegel, D., Galgani, F., and Law, K. L.: A global inventory of small floating plastic debris, Environ. Res. Lett., 10, 124006, https://doi.org/10.1088/1748-9326/10/12/124006, 2015.

Visser, A.: Using random walk models to simulate the vertical distribution of particles in a turbulent water column, Mar. Ecol. Prog. Ser., 158, 275-281, https://doi.org/10.3354/meps158275, 1997.

Wernli, B. H. and Davies, H. C.: A lagrangian-based analysis of extratropical cyclones. I: The method and some applications, Q. J. Roy. Meteor. Soc., 123, 467-489, https://doi.org/10.1002/qj.49712353811, 1997.

Wessel, P. and Smith, W. H. F.: A global, self-consistent, hierarchical, high-resolution shoreline database, J. Geophys. Res., 101, 8741-8743, https://doi.org/10.1029/96JB00104, 1996.

Wettre, C., Johansen, Ø., and Skognes, K.: Development of a 3dimensional oild drift model at dnmi, Norwegian Meteorological Institute, Oslo, Norway, Research Report 133, 50 pp., 2001. 